\title{
Controlling Pest by Integrated Pest Management: A Dynamical Approach
}

\author{
Vandana Kumari \\ Department of Mathematics, \\ Amity Institute of Applied Science, \\ Amity University, Sector-125, Noida, U.P., India. \\ E-mail: vandana_rakesh11@yahoo.com \\ Sudipa Chauhan \\ Department of Mathematics, \\ Amity Institute of Applied Science, \\ Amity University,Sector-125, Noida, U.P., India. \\ Corresponding author: sudipachauhan@gmail.com

\section{Joydip Dhar} \\ Mathematical Modelling and Simulation Laboratory, \\ Atal Bihari Vajpayee Indian Institute of Information Technology and Management, \\ Gwalior, M.P., India. \\ E-mail: jdhar@iiitm.ac.in
}

(Received August 28, 2019; Accepted January 28, 2020)

\begin{abstract}
Integrated Pest Management technique is used to formulate a mathematical model by using biological and chemical control impulsively. The uniform boundedness and the existence of pest extinction and nontrivial equilibrium points is discussed. Further, local stability of pest extinction equilibrium point is studied and it has been derived that if $T \leq T_{\max }$, the pest extinction equilibrium point is locally stable and for $T>T_{\max }$, the system is permanent. It has also been obtained that how delay helps in eradicating pest population more quickly. Finally, analytic results have been validated numerically.
\end{abstract}

Keywords- Plant-pest-natural enemy, Boundedness, Local stability, Permanence.

\section{Introduction}

Plants as we all know conflict between and pests has been a root cause of concern in our ecology from almost two decades. Rescuing crops from predator pests such as insects has become a tedious task for farmers. With the advent in science and technology, effective measures have been discovered to deal with predator pest effectively like introducing natural enemies and chemical pesticides in relevent environment. It is a well known fact that excessive use of chemical pesticide such as organochlorine (DDT and toxaphene) is hazardous both for animals and human being as studied by authors (James, 1997). Therefore, Integrated pest management came into scenario in which selective pesticides control pests as natural predators when regulation through biological means fails. Many biological food web models to control pests have been discussed by many scholars (Changguo et al., 2009; Liu et al., 2013; Jatav et al., 2014; Song et al., 2014) where they took assumptions of either impulsive release of natural enemies or chemical pesticides. Authors (Jatav and Dhar, 2014) studied a model in which they formulated a mathematical model and obtained a threshold value below which pests gets eradicated. Later, many more IPM approach 
International Journal of Mathematical, Engineering and Management Sciences

Vol. 5, No. 4, 769-786, 2020

https://doi.org/10.33889/IJMEMS.2020.5.4.061

inclined models were proposed where impulsive control strategies for pest eradication were introduced and to name a few are (Tang et al., 2005; Akman et al., 2015; El-Shafie, 2018; Paez Chavez et al., 2018). They studied various prospect of IPM method and its application. Scholars (Zhang et al., 2004) did comparison between IPM method and classical method for pest control and obtained that IPM strategy is better than any classical method to control pests. Recently, Yu et al. (2019) introduced IPM method for predator-prey model with Allee effect and stochastic effect respectively where they obtained thresholds based on biological and chemical control. However, in all the papers discussed above no-one discussed significanlty about delays, in particularly gestation delay which in a real situation always exist.

Hence, keeping in mind the above alma matter, we have formulated our model in reference to the previous models and studied the dynamics of the new system with delay. The highlight of the paper is that how delay parameter helps in reducing the pest population more quickly in comparison to the system without delay. The results would be extremely beneficial for those crops where pest population are growing exponentially due to favourable habitable condition. A relevent biological example to our model is as follows:

Australian herb is always at the verge of being attacked by green Lacewing Larvae, which is a well known pest. Encapsulating biological controls like mealy bugs followed by chemical control such as chlorothalonil has shown remarkable results which advocates our approach of hybrid technique. The organisation of the paper is as follows: In Section 2, 3 model formulation and preliminary lemmas are discussed. In Section 4, local stability of pest extinction is achieved followed by permanence in Section 5. Finally, in the last two sections numerical simulation is done for validation of analtical results with conclusion.

\section{Mathematical Model}

We have proposed our mathematical model by the following set of differential equations:

$$
\begin{aligned}
& \frac{d p}{d t}=p(r-p)-a_{1} p q \\
& \frac{d q}{d t}=a_{1} b_{1} p q-a_{2} q(t-\tau) r_{2}(t-\tau) e^{-d_{1} \tau}-D q \\
& \frac{d r_{1}}{d t}=a_{2} b_{2} q(t-\tau) r_{2}(t-\tau) e^{-d_{1} \tau}-\left(D_{3}+\mu_{0}\right) r_{1} \\
& \frac{d r_{2}}{d t}=\mu_{0} r_{1}-D_{3} r_{2} \\
& p\left(t^{+}\right)=p \\
& q\left(t^{+}\right)=(1-\delta) q \\
& r_{1}\left(t^{+}\right)=r_{1}+\mu_{1} \\
& r_{2}\left(t^{+}\right)=r_{2}+\mu_{2}
\end{aligned}
$$

The model completes with the following initial conditions:

$p(\theta)=\phi_{1}(\theta), q(\theta)=\phi_{2}(\theta), r_{1}=\psi_{1}(\theta), r_{2}=\psi_{2}(\theta), \phi_{i}(0)>0, \psi_{i}(0)>0, \theta \in[-\tau, 0]$, $(i=1,2)$, where $\left(\phi_{1}, \phi_{2}, \psi_{1}, \psi_{2}\right) \in C\left([-\tau, 0], \mathbb{R}_{+}^{4}\right.$, the Banach space of continuous functions mapping on the interval $[-\tau, 0]$ into $\mathbb{R}_{+}^{4}$. The graphical representation of the model is 
International Journal of Mathematical, Engineering and Management Sciences

Vol. 5, No. 4, 769-786, 2020

https://doi.org/10.33889/IJMEMS.2020.5.4.061

as follows in Figure 1. Negative and positive sign represents outgoing and incoming rates.

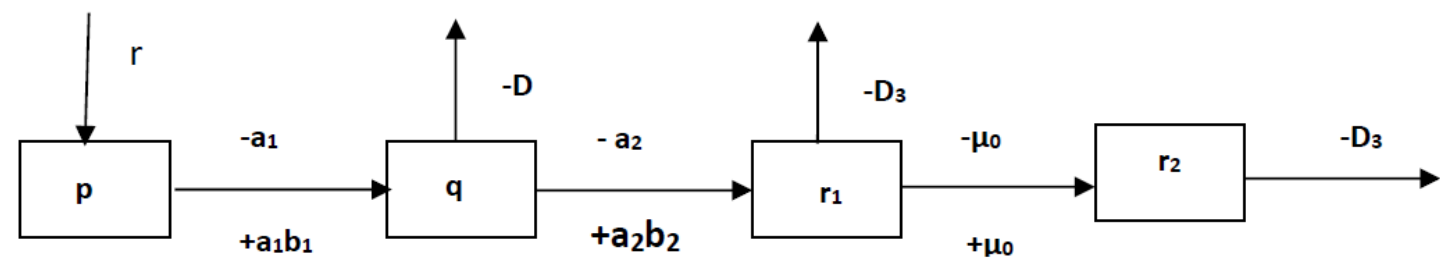

Figure 1. Graphical representation of model

The parameters/variables used in the model are explained in detail in Table 1 mentioned below and for convenience $t$ is removed from the variables throughout the paper.

Table. 1 Meaning of parameters /variables

\begin{tabular}{|c|l|}
\hline Parameters/Variables & Meaning \\
\hline$r_{1}(t)$ & Immature natural enemy \\
\hline$r$ & growth rate of plant population \\
\hline$r_{2}(\mathrm{t})$ & Mature natural enemy \\
\hline$\tau$ & Time delay \\
\hline$p(t)$ & Plant population \\
\hline$a_{1}$ & Rate at which plant population is decreasing to pest population \\
\hline$b_{1}$ & Growth rate of pest population \\
\hline $\mathrm{D}$ & Mortality Rate \\
\hline$a_{2}$ & Rate at which pest population is decreasing \\
\hline$b_{2}$ & Rate at immature natural enemy population \\
\hline$\mu_{0}$ & Mortality rate of immature natural enemy \\
\hline$D_{3}$ & Mortality rate of mature natural enemy \\
\hline$T$ & Period of impulse \\
\hline$\mu_{1}$ & Amount of pulse release of immature natural enemy \\
\hline$\mu_{2}$ & Amount of pulse release of mature natural enemy \\
\hline $0 \leq \delta 1$ & harvesting rate of pest through chemical pesticide \\
\hline$q(t)$ & Pest population \\
\hline
\end{tabular}

\section{Preliminary Lemmas}

In this section, we have given a few Lemmas, which will be useful for our main result.

Lemma 3.1 Let us consider the system

$$
\begin{aligned}
& w^{\prime}(t)=b-c w(t), t \neq n T, \\
& w\left(t^{+}\right)=w(t)+\mu, t=n T, n=1,2,3 \ldots
\end{aligned}
$$

Then the system has a positive periodic solution $\widetilde{w}(t)$ and for any solution $w(t)$ of the system (3),we have,

$$
|w(t)-\widetilde{w}(t)| \rightarrow 0
$$

for $t \rightarrow \infty$, where, for

$$
t \in(n t,(n+1) T], \widetilde{w}(t)=\frac{b}{c}+\frac{\mu \exp (-c(t-n T))}{1-\exp (-c T)} \quad \text { with } \widetilde{w}\left(0^{+}\right)=\frac{b}{c}+\frac{\mu}{1-\exp (-c T)}
$$


International Journal of Mathematical, Engineering and Management Sciences

Vol. 5, No. 4, 769-786, 2020

https://doi.org/10.33889/IJMEMS.2020.5.4.061

The boundedness is given lemma 3.2.

Lemma 3.2 There exists a constant $M>0$ s.t $p(t) \leq M, q(t) \leq M, r_{1}(t) \leq M, r_{2}(t) \leq M$, for $(1-2)$ with $t$ being sufficiently large where

$$
M=\frac{M_{0}}{\widetilde{D}}+\frac{\left(\mu_{1}+\mu_{2}\right) \exp (\bar{D} t)}{\exp (\bar{D} t)-1}>0
$$

Now, we will discuss the pest extinction case and our impulsive system $(1-2)$ reduces to:

$$
\left.\begin{array}{ll}
\frac{d r_{1}(t)}{d t}=-\left(D_{3}+\mu_{0}\right) r_{1}(t) & \\
\frac{d r_{2}(t)}{d t}=\mu_{0} r_{1}(t)-D_{3} r_{2}(t) &
\end{array}\right\} t \neq n T,
$$

For the system $(5-6)$, we integrate it over the interval $(n T,(n+1) T]$, and by means of stroboscopic mapping we get, $r_{1}\left((n+1) T^{+}\right)=\exp \left(-\left(D_{3}+\mu_{0}\right) T\right) r_{1}\left(n T^{+}\right)+\mu_{1}$

Thus the corresponding periodic solution of $(5-6)$ in $t \in(n T,(n+1) T]$ is,

with

$$
\tilde{r}_{1}(t)=\frac{\mu_{1} \exp \left(-\left(D_{3}+\mu_{0}\right)(t-n T)\right)}{1-\exp \left(-\left(D_{3}+\mu_{0}\right) T\right)}
$$

$$
\tilde{r}_{1}\left(0^{+}\right)=\frac{\mu_{1}}{1-\exp \left(-\left(D_{3}+\mu_{0}\right) T\right)}
$$

and is stable globally. Substituting $\tilde{r}_{1}(t)$ into $(5-6)$, we obtain the following subsystem:

$$
\left.\begin{array}{l}
\frac{d r_{2}(t)}{d t}=\mu_{0} \tilde{r}_{1}(t)-D_{3} r_{2}(t), t \neq n T \\
r_{2}\left(t^{+}\right)=r_{2}+\mu_{2}, t=n T
\end{array}\right\}
$$

Further, integrating (7) in the interval $(n T,(n+1) T]$, we get,

$$
\tilde{r}_{2}(t)=\frac{-\mu_{1} \exp \left(-\left(D_{3}+\mu_{0}\right)(t-n T)\right)}{1-\exp \left(-\left(D_{3}+\mu_{0}\right) T\right)}+\frac{\left(\mu_{1}+\mu_{2}\right) \exp \left(-D_{3}(t-n T)\right)}{1-\exp \left(-D_{3} T\right)},
$$

with initial value

$$
\tilde{r}_{2}\left(0^{+}\right)=\frac{-\mu_{1}}{1-\exp \left(-\left(D_{3}+\mu_{0}\right) T\right)}+\frac{\left(\mu_{1}+\mu_{2}\right)}{1-\exp \left(-D_{3} T\right)^{\prime}}
$$


International Journal of Mathematical, Engineering and Management Sciences

Vol. 5, No. 4, 769-786, 2020

https://doi.org/10.33889/IJMEMS.2020.5.4.061

which is stable globally.

Moreover, due to the absence of pest, the subsystem of $(1-2)$ can also be considered as follows:

$$
\frac{d p(t)}{d t}=p(r-p)
$$

With $p=0$ as unstable equilibrium and $p=r$ as globally stable. Therefore, the two periodic solutions of $(1-2)$ are $\left(0,0, \tilde{r}_{1}, \tilde{r}_{2}\right)$ and $\left(r, 0, \tilde{r}_{1}, \tilde{r}_{2}\right)$.

\section{Local Stability of Pest Extinction Case}

This section will discuss the local stability analysis of the equilibrium point with pest population.

Theorem 4.1 Let $\left(p, q, r_{1}, r_{2}\right)$ be a solution of $(1-2)$, Then

(i) $\left(0,0, \tilde{r}_{1}, \tilde{r}_{2}\right)$ is unstable.

(ii) $\left(r, 0, \tilde{r}_{1}, \tilde{r}_{2}\right)$ is locally asymptotically stable iff $T \leq T_{\max }$, where

$$
T_{\text {max }}=\frac{1}{\left(a_{1} b_{1}-d\right)}\left\{\log \frac{1}{(1-\delta)}+e^{-d_{1} \tau} a_{2}\left(\frac{D_{3} \mu_{2}+\mu_{0}\left(\mu_{1}+\mu_{2}\right)}{D_{3}\left(D_{3}+\mu_{0}\right)}\right)\right\}, a_{1} b_{1}>d
$$

Proof: (i) Here, we define,

$$
p=\phi_{1}, q=\phi_{2}, r_{1}=\tilde{r}_{1}+\phi_{3}, r_{2}=\tilde{r}_{2}+\phi_{4}
$$

where, $\phi_{1}(t), \phi_{2}(t), \phi_{3}(t), \phi_{4}(t)$ are perturbation in $p, q, r_{1}, r_{2}$ then the system's linearized form becomes:

$$
\begin{aligned}
& \frac{d \phi_{1}(t)}{d t}=-r \phi_{1}(t) \\
& \frac{d \phi_{2}(t)}{d t}=-\left(D+a_{2} \tilde{r}_{2}(t) e^{-d_{1} \tau}\right) \phi_{2}(t) \\
& \frac{d \phi_{3}(t)}{d t}=a_{2} b_{2} \phi_{2}(t) \tilde{r}_{2}(t) e^{-d_{1} \tau}-\left(D_{3}+\mu_{0}\right) \phi_{3}(t) \\
& \frac{d \phi_{4}(t)}{d t}=\mu_{0} \phi_{3}(t)-D_{3} \phi_{4}(t) \\
& \phi_{1}\left(t^{+}\right)=\phi_{1}(t) \\
& \phi_{2}\left(t^{+}\right)=(1-\delta) \phi_{2}(t) \\
& \phi_{3}\left(t^{+}\right)=\phi_{3}(t)+\mu_{1} \\
& \phi_{4}\left(t^{+}\right)=\phi_{4}(t)+\mu_{2}
\end{aligned}
$$

Let $\phi(t)$ be the fundamental matrix of $(10-11)$, then $\phi(t)$ must satisfy, 
International Journal of Mathematical, Engineering and Management Sciences

Vol. 5, No. 4, 769-786, 2020

https://doi.org/10.33889/IJMEMS.2020.5.4.061

$$
\frac{d \phi(t)}{d t}=\left[\begin{array}{llll}
r & 0 & 0 & 0 \\
0 & -\left(D+a_{2} \tilde{r}_{2}(t) e^{-d_{1} \tau}\right) & 0 & 0 \\
0 & a_{2} b_{2} \tilde{r}_{2}(t) e^{-d_{1} \tau} & -\left(D_{3}+\mu_{0}\right) & 0 \\
0 & 0 & \mu_{0} & -D_{3}
\end{array}\right] \phi(t)=A \phi(t)
$$

Thus, the monodromy matrix of $(10-11)$ is

$$
M=\left[\begin{array}{llll}
1 & 0 & 0 & 0 \\
0 & 1-\delta & 0 & 0 \\
0 & 0 & 1 & 0 \\
0 & 0 & 0 & 1
\end{array}\right] \phi(t)
$$

From (12), we get $\phi(t)=\phi(0) \exp \left(\int_{0}^{T} A d t\right)$, where $\phi(0)$ is an identity matrix and hence the eigen values corresponding to matrix $M$ are as follows:

$$
\begin{gathered}
\lambda_{3}=\exp \left(-\left(D_{3}+\mu_{0}\right)\right) T<1, \quad \lambda_{4}=\exp \left(-D_{3} T\right)<1, \quad \lambda_{1}=\exp (r T)>1, \\
\lambda_{2}=(1-\delta) \exp \int_{0}^{T}\left(-\left(D+a_{2} \tilde{r}_{2}(t) e^{-d_{1} \tau}\right)\right) d t<1 .
\end{gathered}
$$

Therefore, according to the Floquet theory (Bainov and Sineonov, 1993) the pest eradication periodic solution is unstable as $\left|\lambda_{1}\right|>1$.

Remark 1: The effect of delay can be easily seen in the value of $T_{\max }$ which helps in reducing its value.

(ii) The local stability of $\left(r, 0, \tilde{r}_{1}(t), \tilde{r}_{2}(t)\right)$ is proved in the similar fashion. We define $p=r+$ $\phi_{1}(t), q=\phi_{2}(t), r_{1}=\tilde{r}_{1}(t)+\phi_{3}(t), r_{2}=\tilde{r}_{2}(t)+\phi_{4}(t)$ and the system $(1-2)^{\prime} s$ linearized form is as follows:

$$
\begin{aligned}
& \frac{d \phi_{1}(t)}{d t}=-r \phi_{1}(t)-a_{1} \phi_{2} \\
& \frac{d \phi_{2}(t)}{d t}=\left(a_{1} b_{1}-D-a_{2} \tilde{r}_{2}(t) e^{-d_{1} \tau}\right) \phi_{2}(t) \\
& \frac{d \phi_{3}(t)}{d t}=a_{2} b_{2} \phi_{2}(t) \tilde{r}_{2}(t) e^{-d_{1} \tau}-\left(D_{3}+\mu_{0}\right) \phi_{3}(t) \\
& \frac{d \phi_{4}(t)}{d t}=\mu_{0} \phi_{3}(t)-D_{3} \phi_{4}(t) \\
& \phi_{1}\left(t^{+}\right)=\phi_{1}(t), \\
& \phi_{2}\left(t^{+}\right)=(1-\delta) \phi_{2}(t) \\
& \phi_{3}\left(t^{+}\right)=\phi_{3}(t)+\mu_{1} \\
& \phi_{4}\left(t^{+}\right)=\phi_{4}(t)+\mu_{2}
\end{aligned}
$$


Let $\phi(t)$ be the fundamental matrix of $(13-14)$, then $\phi(t)$ must satisfy

$$
\begin{aligned}
\frac{d \phi(t)}{d t}= & =\left[\begin{array}{llll}
-r & -a_{1} & 0 & 0 \\
0 & a_{1} b_{1}-D-a_{2} \tilde{r}_{2}(t) e^{-d_{1} \tau} & 0 & 0 \\
0 & a_{2} b_{2} \tilde{r}_{2}(t) e^{-d_{1} \tau} & -\left(D_{3}+\mu_{0}\right) & 0 \\
0 & 0 & \mu_{0} & -D_{3}
\end{array}\right] \phi(t) \\
\frac{d \phi(t)}{d t} & =A \phi(t)
\end{aligned}
$$

Thus, the monodromy matrix of $(13-14)$ is

$$
M=\left[\begin{array}{llll}
1 & 0 & 0 & 0 \\
0 & 1-\delta & 0 & 0 \\
0 & 0 & 1 & 0 \\
0 & 0 & 0 & 1
\end{array}\right] \phi(t)
$$

From (15), we get $\phi(t)=\phi(0) \exp \left(\int_{0}^{T} A d t\right)$, where $\phi(0)$ is an identity matrix. Then the characteristic values obtained for $M$ are as follows:

$$
\begin{gathered}
\lambda_{1}=\exp (-r T)<1, \quad \lambda_{2}=(1-\delta) \exp \int_{0}^{T}\left(a_{1} b_{1}-D-a_{2} \tilde{r}_{2}(t) e^{-d_{1} \tau}\right)<1, \\
\lambda_{3}=\exp \left(\left(-\left(D_{3}+\mu_{0}\right)-\lambda\right) T\right)<1, \quad \lambda_{4}=\exp \left(-D_{3} T\right)<1 .
\end{gathered}
$$

Therefore, pest eradication periodic solution of $(1-2)$ is locally asymptotically stable as per Floquet theory (Bainov and Sineonov, 1993) if and only if $\left|\lambda_{2}\right| \leq 1$ which implies $T \leq T_{\max }$. Hence, the theorem is proved.

\section{Permanence}

In this section, we will discuss permanence of system $(1-2)$.

Theorem 5.1 The system (1-2) is permanent if $T>T_{\max }$.

Proof. Suppose $\left(p, q, r_{1}, r_{2}\right)$ is the solution of the system $(1-2), t$ being removed for convenience, We have already proved that $p(t) \leq M, q(t) \leq M, r_{1}(t) \leq M$ and $r_{2}(t) \leq M \forall$ $t$. From, $(1-2)$ we have $\frac{d p}{d t} \geq p\left(r-a_{1} M-p\right) \quad$ which implies that $p(t)>r-a_{1} M \triangleq m_{1}$ for all large $t$. For small $\epsilon_{4}>0$, we choose $m_{1}=1-\epsilon>0$ and also define,

$$
m_{2}=\frac{-\mu_{1} \exp \left(-\left(D_{3}+\mu_{0}\right)(t-n T)\right)}{1-\exp \left(-\left(D_{3}+\mu_{0}\right) T\right)}-\epsilon_{4}>0
$$


International Journal of Mathematical, Engineering and Management Sciences

Vol. 5, No. 4, 769-786, 2020

https://doi.org/10.33889/IJMEMS.2020.5.4.061

$m_{3}=\frac{-\mu_{1} \exp \left(-\left(D_{3}+\mu_{0}\right)(t-n T)\right)}{1-\exp \left(-\left(D_{3}+\mu_{0}\right) T\right)}+\frac{\left(\mu_{1}+\mu_{2}\right) \exp \left(-D_{3}(t-n T)\right)}{1-\exp \left(-D_{3} T\right)}-\frac{\epsilon_{4} \mu_{0}}{D_{3}}-\epsilon_{4}>0$.

Now, the system $(1-2)$ can be rewritten as:

$$
\left.\begin{array}{ll}
\frac{d r_{1}(t)}{d t}=-\left(D_{3}+\mu_{0}\right) r_{1}(t) & \\
\frac{d r_{2}(t)}{d t}=\mu_{0} r_{1}(t)-D_{3} r_{2}(t) &
\end{array}\right\} t \neq n T,
$$

The system $(16-17)$ is same as $(5-6)$, using same technique, we can easily find that $r_{1}(t)>$ $m_{2}$ and $r_{2}(t)>m_{3} \forall t$. Hence, for proving the permanence we have only have to prove $m_{4}>$ 0 , such that $q(t) \geq m_{4} \forall t$ which will be done in two steps.

Step 1: Let $q(t) \geq m_{4}$ is false $\exists$ a $t_{1} \in(0, \infty)$ s.t $q(t)<m_{4} \quad \forall \quad t>t_{1}$. Using this supposition, we get subsystem of $(1-2)$ :

$$
\begin{gathered}
\frac{d r_{1}(t)}{d t} \leq a_{2} b_{2} M m_{4} e^{-d_{1} \tau}-\left(D_{3}+\mu_{0}\right) r_{1}, t \neq n T \\
r_{1}\left(t^{+}\right)=r_{1}(t)+\mu_{1}, t=n T, n=1,2,3 \ldots \ldots
\end{gathered}
$$

Let us assume the comparison system:

$$
\left.\begin{array}{l}
\frac{d \bar{w}_{1}(t)}{d t} \leq a_{2} b_{2} M m_{4} e^{-d_{1} \tau}-\left(D_{3}+\mu_{0}\right) \bar{w}_{1}(t), t \neq n T \\
\bar{w}_{1}\left(t^{+}\right)=\bar{w}_{1}(t)+\mu_{1}, t=n T, n=1,2,3 \ldots
\end{array}\right\}
$$

Using lemma 3.1, equation (18) has periodic solution

$$
(t) \widetilde{w}_{1}=\frac{a_{2} b_{2} m_{4} M \exp \left(-d_{1} \tau\right)}{D_{3}+\mu_{0}}+\frac{\mu_{1} \exp \left(-\left(D_{3}+\mu_{0}\right)(t-n T)\right)}{1-\exp \left(-\left(D_{3}+\mu_{0}\right) T\right)}
$$

which is globally asymptotically stable. Then, $\exists$ an $\epsilon_{5}>0$ s.t

$$
r_{1}(t) \leq \widetilde{w}_{1}(t)<\frac{a_{2} b_{2} m_{4} M \exp \left(-d_{1} \tau\right)}{D_{3}+\mu_{0}}+\frac{\mu_{1} \exp \left(-\left(D_{3}+\mu_{0}\right)(t-n T)\right)}{1-\exp \left(-\left(D_{3}+\mu_{0}\right) T\right)}+\epsilon_{5}>0 .
$$

For sufficiently large $t$. Thus we find the following subsystem of $(1-2)$ : 
International Journal of Mathematical, Engineering and Management Sciences

Vol. 5, No. 4, 769-786, 2020

https://doi.org/10.33889/IJMEMS.2020.5.4.061

$$
\left.\begin{array}{l}
\frac{d r_{2}(t)}{d t}=\mu_{0}\left(\frac{a_{2} b_{2} m_{4} M \exp \left(-d_{1} \tau\right)}{D_{3}+\mu_{0}}+\frac{\mu_{1} \exp \left(-\left(D_{3}+\mu_{0}\right)(t-n T)\right)}{1-\exp \left(-\left(D_{3}+\mu_{0}\right) T\right)}+\epsilon_{5}\right)-D_{3} r_{2}, t \neq n T \\
r_{2}\left(t^{+}\right)=r_{2}+\mu_{2}, \quad t=n T, \quad n=1,2,3 \ldots \ldots
\end{array}\right\}
$$

Consider the comparison system (19) as follows:

$$
\left.\begin{array}{l}
\frac{d \bar{w} \quad{ }_{2}(t)}{d t}=\mu_{0}\left(\frac{a_{2} b_{2} m_{4} M \exp \left(-d_{1} \tau\right)}{D_{3}+\mu_{0}}+\frac{\mu_{1} \exp \left(-\left(D_{3}+\mu_{0}\right)(t-n T)\right)}{1-\exp \left(-\left(D_{3}+\mu_{0}\right) T\right)}+\epsilon_{5}\right)(t)-D_{3} \bar{w}_{2}(t), t \neq n T \\
\bar{w}_{2}\left(t^{+}\right)=\bar{w}_{2}(t)+\mu_{2}, t=n T, n=1,2,3 \ldots \ldots .
\end{array}\right\}
$$

Similarly, system (20) also has a periodic solution

$$
\begin{array}{r}
r_{2}(t)<\widetilde{w}_{2}(t)<\frac{-\mu_{1} \exp \left(-\left(D_{3}+\mu_{0}\right)(t-n T)\right)}{1-\exp \left(-\left(D_{3}+\mu_{0}\right) T\right)}+\frac{\left(\mu_{1}+\mu_{2}\right) \exp \left(-D_{3}(t-n T)\right)}{1-\exp \left(-D_{3} T\right)} \\
+\frac{\mu_{0}}{D_{3}}\left(\frac{a_{2} b_{2} m_{4} M \exp \left(-d_{1} \tau\right)}{\left.D_{3}+\mu_{0}\right)}+\epsilon_{5}\right) \\
\widetilde{\widetilde{w}}_{2}(t)<\frac{-\mu_{1} \exp \left(-\left(D_{3}+\mu_{0}\right)(t-n T)\right)}{1-\exp \left(-\left(D_{3}+\mu_{0}\right) T\right)}+\frac{\left(\mu_{1}+\mu_{2}\right) \exp \left(-D_{3}(t-n T)\right)}{1-\exp \left(-D_{3} T\right)} \\
+\frac{\mu_{0}}{D_{3}}\left(\frac{a_{2} b_{2} m_{4} M \exp \left(-d_{1} \tau\right)}{\left(D_{3}+\mu_{0}\right)} \epsilon_{5}\right)
\end{array}
$$

which is globally asymptotically stable and $\exists$ an $\epsilon_{6}>0$ s.t

$$
\begin{gathered}
r_{2}(t)<{\widetilde{w_{2}}}_{2}(t)<\frac{-\mu_{1} \exp \left(-\left(D_{3}+\mu_{0}\right)(t-n T)\right)}{1-\exp \left(-\left(D_{3}+\mu_{0}\right) T\right)}+\frac{\left(\mu_{1}+\mu_{2}\right) \exp \left(-D_{3}(t-n T)\right)}{1-\exp \left(-D_{3} T\right)} \\
+\frac{\mu_{0}}{D_{3}}\left(\frac{a_{2} b_{2} m_{4} M \exp \left(-d_{1} \tau\right)}{\left(D_{3}+\mu_{0}\right)}+\epsilon_{5}\right)+\epsilon_{6} .
\end{gathered}
$$

It shows that $\exists$ a $T_{1}>0$ s.t for $n T<t \leq(n+1) T$, we are having the following subsystem of $(1-2)$ :

$$
\left.\begin{array}{l}
\frac{d q(t)}{d t} \geq\left[a_{1} b_{1} m_{1}-a_{2}\left(\widetilde{w}_{2}(t)+\epsilon_{6}\right) e^{-d_{1} \tau}-D\right] q, t \neq n T \\
q\left(t^{+}\right)=(1-\delta) q(t), t=n T, \text { and }, t>T_{1}
\end{array}\right\}
$$

Integrating the system, (22) on $(n T,(n+1) T], n \geq N_{1}$ (here, $N_{1}$ is the nonnegative integer and $N_{1} T \geq T_{1}$ ), then we obtain that, 
International Journal of Mathematical, Engineering and Management Sciences

Vol. 5, No. 4, 769-786, 2020

https://doi.org/10.33889/IJMEMS.2020.5.4.061

$$
\begin{aligned}
q((n+1) T) & \geq(1-\delta) q\left(n T^{+}\right) \exp \left(\int_{n T}^{(n+1) T}\left(a_{1} b_{1} m_{1}-a_{2}\left(\tilde{\bar{r}}_{2}(t)-\epsilon_{6}\right) e^{-d_{1} \tau}-D\right) d t\right) \\
= & q\left(n T^{+}\right) \bar{\sigma}
\end{aligned}
$$

where, $\quad \bar{\sigma}(1-\delta) q\left(n T^{+}\right) \exp \left(\int_{n T}^{(n+1) T}\left(a_{1} b_{1} m_{1}-a_{2}\left(\tilde{\tilde{r}}_{2}(t)-\epsilon_{6}\right) e^{-d_{1} \tau}-D\right) d t\right)>1$, as, $T>$ $T_{\text {max }}$, therefore, for $\epsilon_{5}>0$, we obtain that,

$$
\begin{gathered}
\left(a_{1} b_{1} m_{1}-a_{2} \epsilon_{6} \exp \left(-d_{1} \tau\right)-D\right) T-\frac{a_{2} \mu_{0} \exp \left(-d_{1} \tau\right)}{D_{3}}\left(\frac{b_{2} m_{4} M \exp \left(-d_{1} \tau\right)}{\left(D_{3}+\mu_{0}\right)}-\epsilon_{5}\right)- \\
a_{2}\left(\frac{\mu_{1}}{\left(D_{3}+\mu_{0}\right)} \frac{\left(\mu_{1}+\mu_{2}\right) \exp \left(-d_{1} \tau\right)}{D_{3}}\right)-\log \left(\frac{1}{1-\delta}\right)>1 .
\end{gathered}
$$

Thus, $q\left(\left(N_{1}+k\right) T\right) \geq q\left(N_{1} T^{+}\right) \bar{\sigma}^{k} \rightarrow \infty$ as $k \rightarrow \infty$, which violoates our assumption $q(t)<$ $m_{4}$, for every $t>t_{2}$. Hence there exists a $t_{2}>t_{1}$ s.t $q\left(t_{2}\right) \geq m_{4}$.

Step 2: If $q(t) \geq m_{4} \forall \quad t \geq t_{2}$, then our aim will be fulfilled. On the contrary let us assume that $q(t)<m_{4}$ for some $t>t_{2}$. Let $t^{*}=\inf \left\{t \mid q(t)<m_{4}, t>t_{2}\right\}$, then there will be two cases:

Case 1: Let $t^{*}=n_{1} T, n_{1} \in Z^{+}$. In this case $q(t) \geq m_{4}$ for $t \in\left[t_{2}, t^{*}\right)$ and $(1-\delta) m_{4} \leq$ $q\left(t^{*+}=(1-\delta) q\left(t^{*}\right)<m_{4}\right)$. Let $T_{2}=n_{2} T+n_{3} T$, where $n_{2}=n_{2}^{\prime}+n_{2}^{\prime \prime}, n_{2}^{\prime}, n_{2}^{\prime \prime}$ and $n_{3}$ satisfy these inequalities:

$$
\begin{gathered}
n_{2}{ }^{\prime} T>-\frac{1}{D_{3}+\mu_{0}} \ln \frac{\epsilon_{5}}{M+\mu_{1}}, \\
n_{2}{ }^{\prime \prime} T>-\frac{1}{D_{3}+\mu_{0}} \ln \frac{\epsilon_{6}}{M+\mu_{2}}, \\
(1-\delta)^{n_{2}+n_{3}} \exp \left(\eta n_{2} T\right) \exp \left(n_{3} \sigma\right)>1,
\end{gathered}
$$

$\eta=a_{1} b_{1} m_{1}-a_{2} \epsilon_{6} \exp \left(-d_{1} \tau\right)-D<0$. Now, we claim that $\exists$ a time $t_{2}^{\prime} \in\left(t^{*}, t^{*}+T_{2}\right)$ such that $q\left(t_{2}^{\prime}\right) \geq m_{4}$, if it is not true, then $q\left(t_{2}^{\prime}\right)<m_{4}, t_{2}^{\prime} \in\left(t^{*}, t^{*}+T_{2}\right)$. If the system (18) is taken with initial value $\bar{w}_{1}\left(t^{*+}\right)=r_{1}\left(t^{*+}\right)$, then from lemma (3.1) for $t \in(n T,(n+1) T]$, we have

$\bar{w}_{1}(t)=\left(\bar{w}_{1}\left(t^{*+}\right)-\frac{a_{2} b_{2} m_{4} M \exp \left(-d_{1} \tau\right)}{D_{3}+\mu_{0}}+\frac{\mu_{1}}{1-\exp \left(-\left(D_{3}+\mu_{0}\right) T\right)}\right) \exp \left(-\left(D_{3}+\mu_{0}\right)\left(t-t^{*}\right)\right)+\widetilde{\widetilde{W}}_{1}(t)$, for $n_{1} \leq n \leq n_{1}+n_{2}+n_{3}$ which shows that $\left|\bar{w}_{1}(t)-\widetilde{\widetilde{w}}_{1}(t)\right| \leq\left(M+\mu_{1}\right) \exp \left(-\left(D_{3}+\right.\right.$ $\left.\left.\mu_{0}\right)\left(t-n_{1} T\right)\right)<\epsilon_{5}$, and $r_{1}(t) \leq \bar{w}_{1}(t)<\widetilde{w}_{1}(t)+\epsilon_{5}$ for $t^{*}+n_{2}^{\prime} T \leq t \leq t^{*}+T_{2}$.

Now, from the system (18) with initial values $\bar{w}_{2}\left(t^{*}+n_{2}^{\prime} T\right)=q_{2}\left(t^{*}+n_{2}^{\prime} T\right) \geq 0$ and again from lemma (3.1), we have $\left|\bar{w}_{1}(t)-\widetilde{\widetilde{w}}_{1}(t)\right|<\left(M+\mu_{2}\right) \exp \left(D_{3}\left(t-\left(n_{1}+N_{2}^{\prime}\right) T\right)\right)<$ $\epsilon_{6}$, and $r_{2}(t) \leq \bar{w}_{2}(t)<\widetilde{w}_{2}(t)+\epsilon_{6}$ for $t^{*}+n_{2}^{\prime} T+n_{2}^{\prime \prime} T \leq t \leq t^{*}+T_{2}$, which shows that 
International Journal of Mathematical, Engineering and Management Sciences

Vol. 5, No. 4, 769-786, 2020

https://doi.org/10.33889/IJMEMS.2020.5.4.061

system (22) holds for $\left[t^{*}+n_{2} T, t^{*}+T_{2}\right]$.

Integrating equation (22) on $\left[t^{*}+n_{2} T, t^{*}+T_{2}\right]$, we have

$$
q\left(\left(n_{1}+n_{2}+n_{3}\right) T\right) \geq q\left(\left(n_{1}+n_{2}\right) T\right)(1-\delta)^{n_{3}} \exp \left(n_{3} \sigma\right)
$$

In addition from the system $(1-2)$, we have

$$
\left.\begin{array}{rl}
\frac{d q(t)}{d t} & =\left(a_{1} b_{1} m_{1}-a_{2} M e^{-d_{1} \tau}-D\right) q(t)=\eta q(t), t \neq n T \\
q\left(t^{+}\right) & =(1-\delta) q, t=n T, n=1,2,3 \ldots
\end{array}\right\}
$$

On integrating (24) in the interval $\left[T^{*},\left(n_{1}+n_{2}\right) T\right]$, it is obtained that

$$
q\left(\left(n_{1}+n_{2}\right) T\right) \geq m_{4}(1-\delta)^{n_{2}} \exp \left(\eta n_{2} T\right)
$$

Now substitute (25) into (24), we get that

$$
q\left(\left(n_{1}+n_{2}+n_{3}\right) T\right) \geq m_{4}(1-\delta)^{n_{2}+n_{3}} \exp \left(n_{3} \sigma\right) \exp \left(\eta n_{2} T\right)>m_{4}
$$

which contradicts to our supposition, so there exists a time $t_{2}^{\prime} \in\left[t^{*}, t^{*}+T_{2}\right]$ such that $q_{2}^{\prime} \geq$ $m_{4}$. Let $\hat{t}=\inf \left\{t \mid t \geq t^{*}, q(t) \geq m_{4}\right\} \quad$,since $\quad 0<\delta<1, q\left(n T^{+}\right)=(1-\delta) q(n T)<$ $q(n T)$ and $q(t)<m_{4}, t \in\left(t^{*}, \hat{t}\right)$. Thus, $q(\hat{t})=m_{4}$.

Supposet $\in\left(t^{*}+(l-1) T, T^{*}+l T\right]$ (lis a positive integer) and $l \leq n_{2}+n_{3}$, from the system (24), we have

$$
\begin{gathered}
q(t) \geq q\left(t^{*}+(l-1) T\right) \exp \left(\eta\left(t-t^{*}-(l-1)\right) T\right) \\
q(t) \geq q\left(n T^{+}\right) \exp (\eta T(l-1))(1-\delta)^{l-1} \exp (\eta T) \\
q(t) \geq m_{4}(1-\delta)^{l} \exp (l \eta T) \\
\left.q(t) \geq m_{4}(1-\delta)^{(} n_{2}+n_{3}\right) \exp \left(\left(n_{2}+n_{3}\right) \eta T\right) \triangleq \bar{m}_{4}
\end{gathered}
$$

for $t>\hat{t}$. The same argument can be continued since $q(\hat{t}) \geq m_{4}$. Hence $q(t) \geq \bar{m}_{4} \forall t>t_{2}$.

Case 2: If $t^{*} \neq n T$, then $q\left(t^{*}\right)=m_{4}$ and $q(t) \geq m_{4}, t \in\left[t_{2}, t^{*}\right]$. Suppose $t^{*} \in\left(n_{1}^{\prime} T,\left(n_{1}^{\prime}+\right.\right.$ 1)T], we are having two subcases for $t \in\left[t^{*},\left(n_{1}^{\prime}+1\right) T\right]$ as given below:

Case a: $\quad q(t) \leq m_{4}, t \epsilon\left[t^{*},\left(n_{1}^{\prime}+1\right) T\right]$, we claim that there exists a $t_{3} \varepsilon\left[\left(n_{1}^{\prime}+1\right) T,\left(n_{1}^{\prime}+\right.\right.$ 1)T $\left.T+T_{2}\right]$ s.t $q\left(t_{3}\right)>m_{4}$. Otherwise, integrating system (24) on the interval $\left[\left(n_{1}^{\prime}+1+\right.\right.$ $\left.\left.n_{2}\right) T,\left(n_{1}^{\prime}+1+n_{2}+n_{3}\right) T\right]$, we have, $q\left(\left(n_{1}^{\prime}+1+n_{2}+n_{3}\right) T\right) \geq q\left(\left(n_{1}^{\prime}+1+n_{2}\right) T\right)(1-$ $\delta)^{n_{3}} \exp \left(n_{3} \sigma\right)$

Since $q(t) \leq m_{4}, t \in\left[t^{*},\left(n_{1}^{\prime}+1\right) T\right]$, therefore, (13) holds on $\left[t^{*},\left(n_{1}^{\prime}+n_{2}+n_{3}\right) T\right]$. 
International Journal of Mathematical, Engineering and Management Sciences

Vol. 5, No. 4, 769-786, 2020

https://doi.org/10.33889/IJMEMS.2020.5.4.061

Thus,

and

$$
\begin{gathered}
q\left(\left(n_{1}^{\prime}+1+n_{2}\right) T\right)=q\left(t^{*}\right)(1-\delta)^{n_{2}} \exp \left(\eta\left(n_{1}^{\prime}+1+n_{2}\right) T-t^{*}\right) \\
q\left(\left(n_{1}^{\prime}+1+n_{2}\right) T\right) \geq m_{4}(1-\delta)^{n_{2}} \exp \left(\eta n_{2} T\right)
\end{gathered}
$$

$$
q\left(\left(n_{1}^{\prime}+1+n_{2}+n_{3}\right) T\right) \geq m_{4}(1-\delta)^{n_{2}+n_{3}} \exp \left(\eta n_{2} T\right) \exp \left(n_{3} \sigma\right)>m_{4}
$$

which negates the assumption. Let $\breve{t}=\inf \left\{t \mid q \geq m_{4}, t>t^{*}\right\}$, then $q(\breve{t})=m_{4}$ and $q<m_{4}, t \in$ $\left(t^{*}, \breve{t}\right)$. Choose $t \in\left(n_{1}^{\prime} T+\left(l^{\prime}-1\right) T, n_{1}^{\prime} T+l^{\prime} T\right] \subset\left(t^{*}, \breve{t}\right), l^{\prime}$ is a positive integer and $l^{\prime}<1+$ $n_{2}+n_{3}$, we have

$$
\begin{gathered}
q(t) \geq q\left(\left(n_{1}^{\prime}+l^{\prime}-1\right) T^{+}\right) \exp \left(\eta\left(t-\left(n_{1}^{\prime}+l^{\prime}-1\right) T\right)\right) \\
q(t) \geq(1-\delta)^{l^{\prime}-1} q\left(t^{*}\right) \exp \left(\eta\left(t-t^{*}\right)\right) \\
q(t) \geq m_{4}(1-\delta)^{n_{2}+n_{3}} \exp \left(\eta\left(n_{2}+n_{3}+1\right) T\right) .
\end{gathered}
$$

Hence, $q \geq \bar{m}_{4}$ for $t \in\left(t^{*}, \breve{t}\right)$. For $t>\breve{t}$, we can proceed in the same manner since $q(\breve{t}) \geq m_{4}$.

Case b: If $\exists$ a $t \in\left(t^{*},\left(n_{1}^{\prime}+1\right) T\right)$ s.t $q(t) \geq m_{4}$. Let $\breve{t}=\inf \left(t \mid q(t) \geq m_{4}, t>t^{*}\right)$, then $q(t)<m_{4}$ for $t \in\left[t^{*}, \bar{t}\right)$ and $q(\bar{t})=m_{4}$. For $t \in\left[t^{*}, \bar{t}\right)$ (24) holds. On integrating (24) on $t^{*}, \breve{t}$, we obtain

$$
q \geq q\left(t^{*}\right) \geq \exp \left(\eta\left(t-t^{*}\right) \geq m_{4} \exp (\eta T)>\bar{m}_{4}\right.
$$

Since, $q(\hat{t}) \geq m_{4}$ for $t>\hat{t}$, we can proceed in the same manner. Hence, we have $q(t) \geq \bar{m}_{4}$ for all $t>t_{2}$. Therefore we can conclude that $q(t) \geq \bar{m}_{4}$ for all $t \geq t_{2}$ in both cases.

\section{Numerical Section}

For the intended process, we have taken data per week in view of the short term life cycle of the insect population under investigation. Our aim is to validate the analytical results numerically. We have considered numerical values for the following set of parameters in reference to (Jatav and Dhar, 2014) as mentioned in Table 2.

Table 2. Parametric values

\begin{tabular}{|l|c|c|c|c|c|c|c|c|c|c|}
\hline Parameters & $\mu_{0}$ & $\mathrm{r}$ & $\mathrm{a}_{1}$ & $\mathrm{~b}_{1}$ & $\mathrm{~d}_{1}$ & $\tau$ & $\mathrm{a}_{2}$ & $\mathrm{~b}_{2}$ & $\mathrm{D}$ & $\mathrm{D}_{3}$ \\
\hline Values & 50 & 1 & 1 & 0.1 & 0.3 & 0.2 & 0.3 & 0.5 & 0.03 & 25 \\
\hline
\end{tabular}

Using the above parametric values, we obtained the threshold value $T_{\max }$ for the parameters per week as 0.8 . It is proved that $\left(r, 0, \tilde{r}_{1}(t), \tilde{r}_{1}(t)\right)$ is locally asymptotically stable if $T=0.5<$ $T_{\max }$ as stated above in the theorem 4.1 (Figure 2-5). Further, it is also verified that the system $(A-B)$ is permanent if $T=4>T_{\max }$ (Figure 6-9) which is inline with theorem 5.1. It is also shown that if there is no biological control, that is, $\mu_{1}=0$ and $\mu_{2}=0, \mu_{1}=0$ and $\mu_{2}>$ 0 or $\mu_{1}>0$ and $\mu_{2}=0$, then both plants and pest population survives. This concludes, that solely using chemical pesticide cannot eradicate pest population (Figure 10-14). 
International Journal of Mathematical, Engineering and Management Sciences

Vol. 5, No. 4, 769-786, 2020

https://doi.org/10.33889/IJMEMS.2020.5.4.061

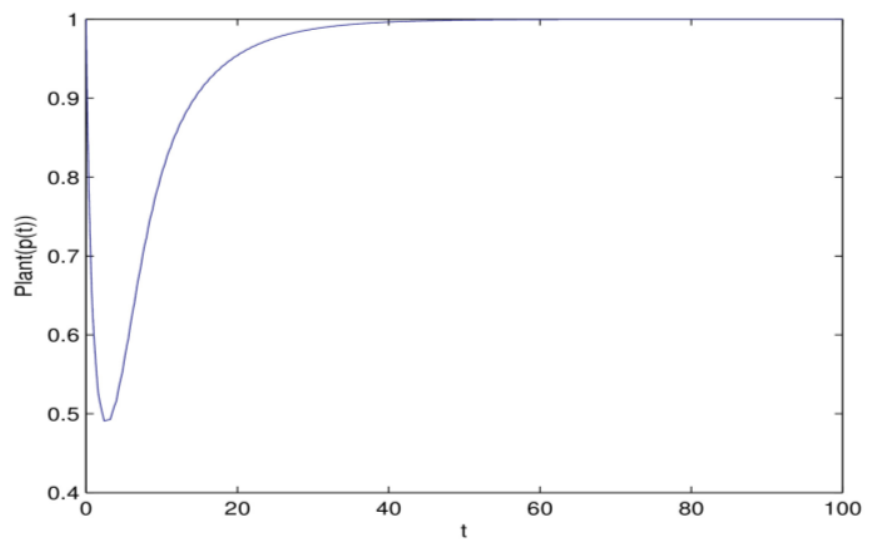

Figure 2. Plant population $(P(t))$ existing

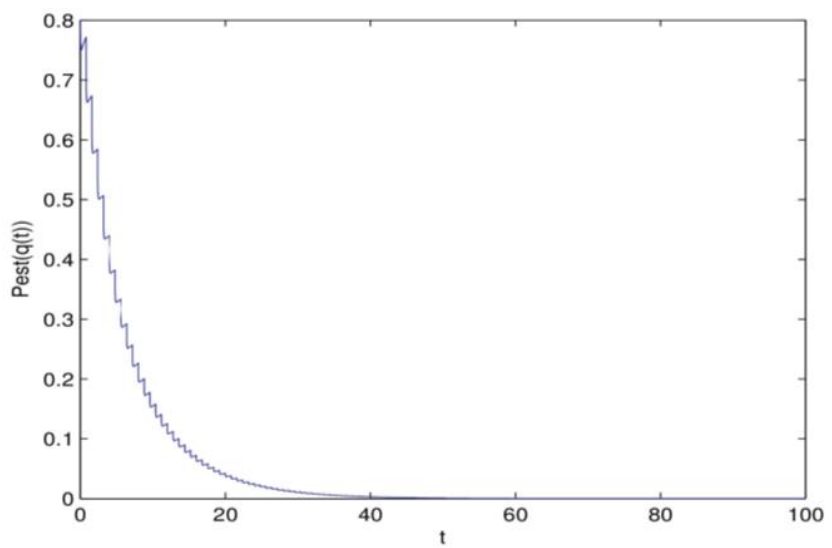

Figure 3. Pest population $(q(t))$ vanishes

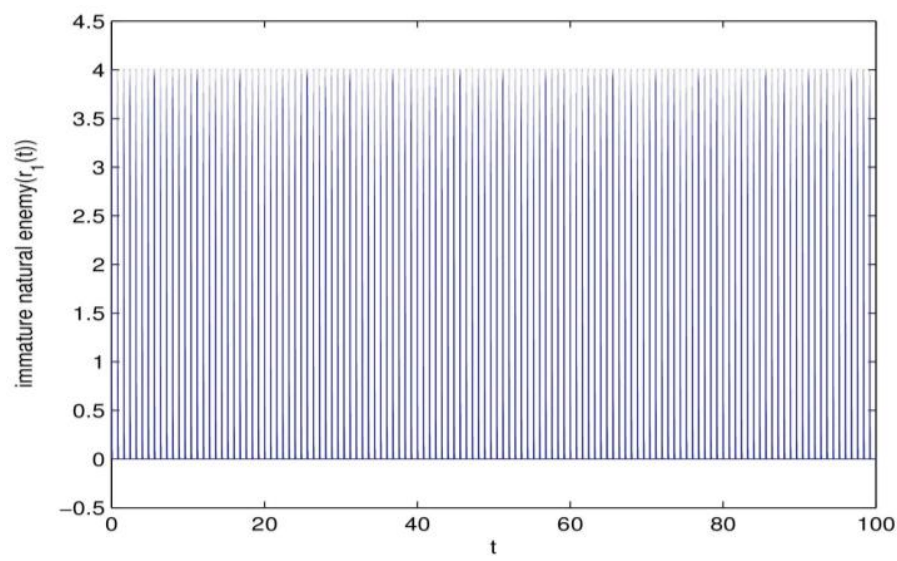

Figure 4. Periodic behaviour of $r_{1}(t)$ 
International Journal of Mathematical, Engineering and Management Sciences

Vol. 5, No. 4, 769-786, 2020

https://doi.org/10.33889/IJMEMS.2020.5.4.061

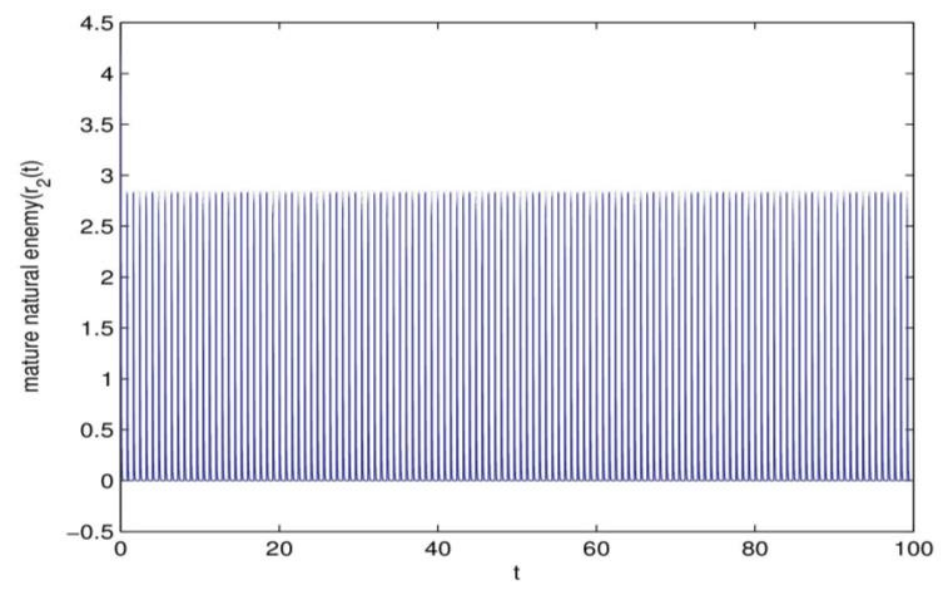

Figure 5. Periodic behaviour of $\left(r_{2}(t)\right)$

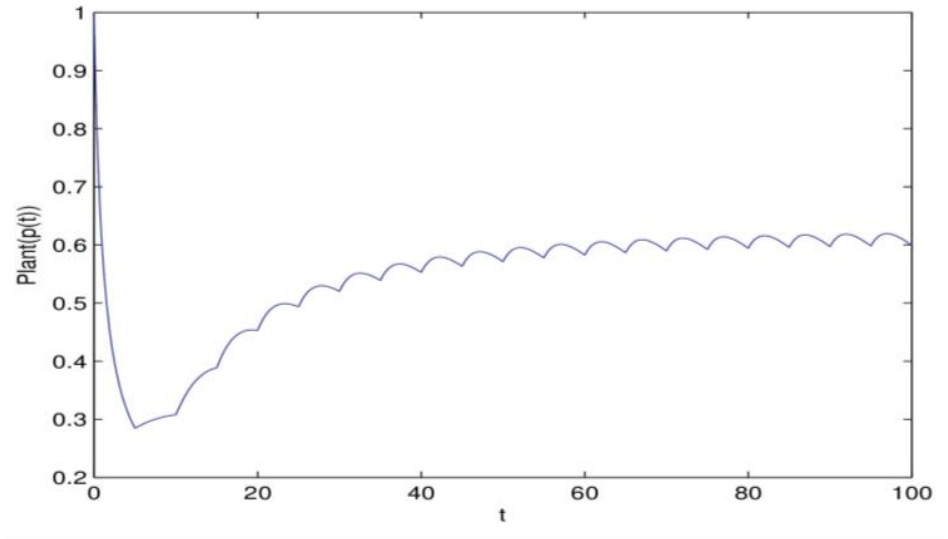

Figure 6. Plant population $(p(t))$ exists

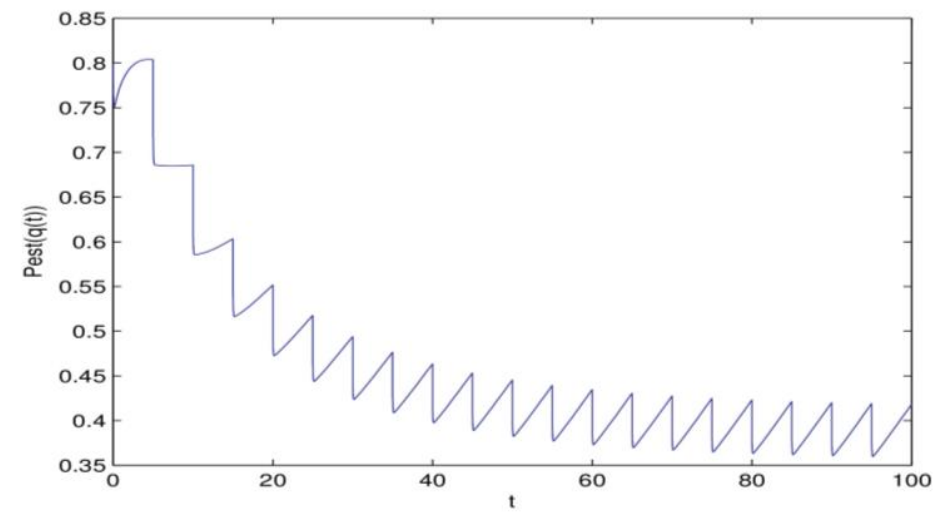

Figure 7. Pest population $(q(t))$ survives 
International Journal of Mathematical, Engineering and Management Sciences

Vol. 5, No. 4, 769-786, 2020

https://doi.org/10.33889/IJMEMS.2020.5.4.061

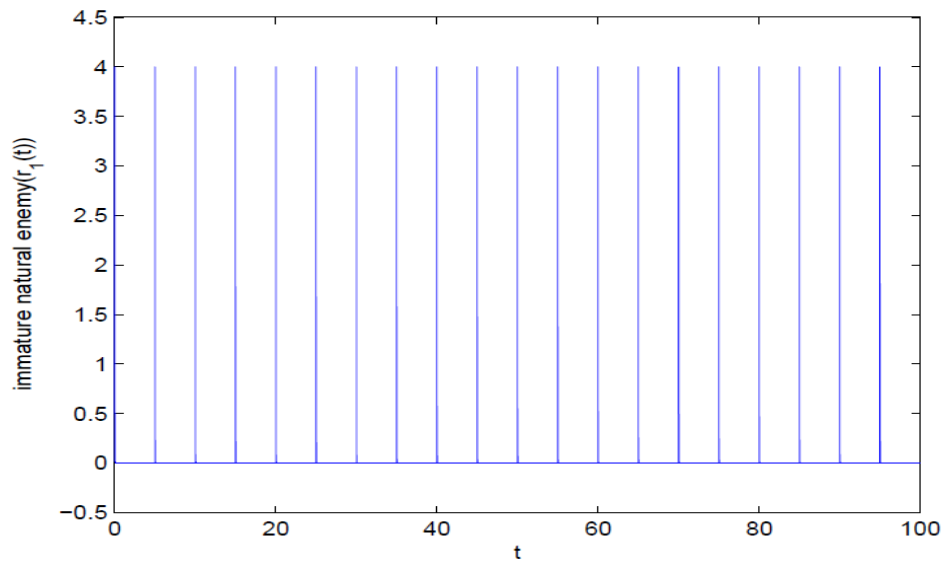

Figure 8. Immature natural enemies $\left(r_{1}(t)\right)$ exists

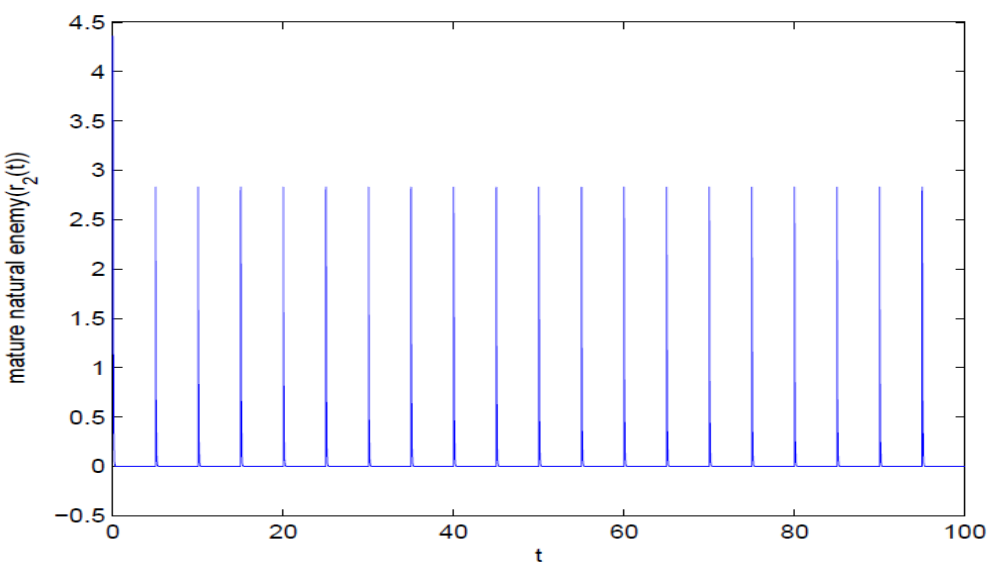

Figure 9. Behaviour of mature natural enemies $\left(r_{2}(t)\right)$

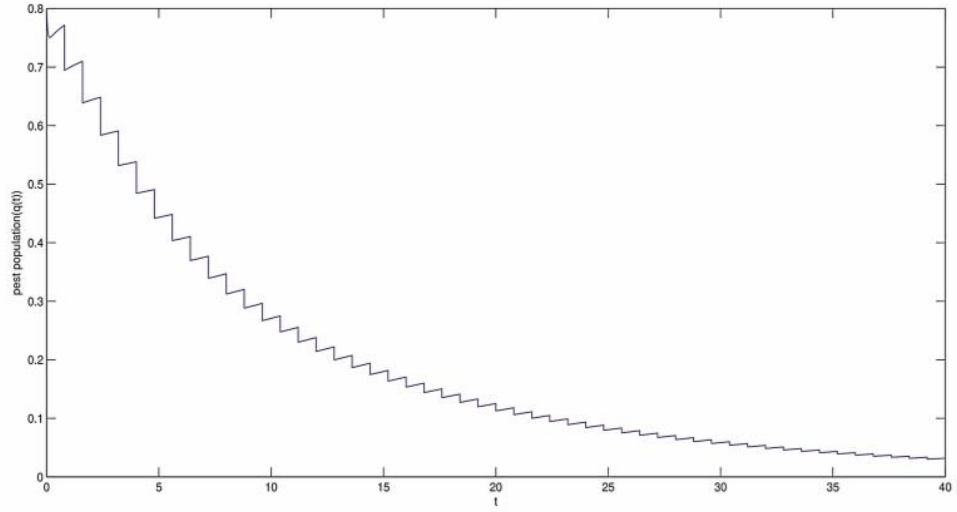

Figure 10. Existence of the pest population $(q(t))$ for $\mu_{1}, \mu_{2}=0$ 
International Journal of Mathematical, Engineering and Management Sciences

Vol. 5, No. 4, 769-786, 2020

https://doi.org/10.33889/IJMEMS.2020.5.4.061

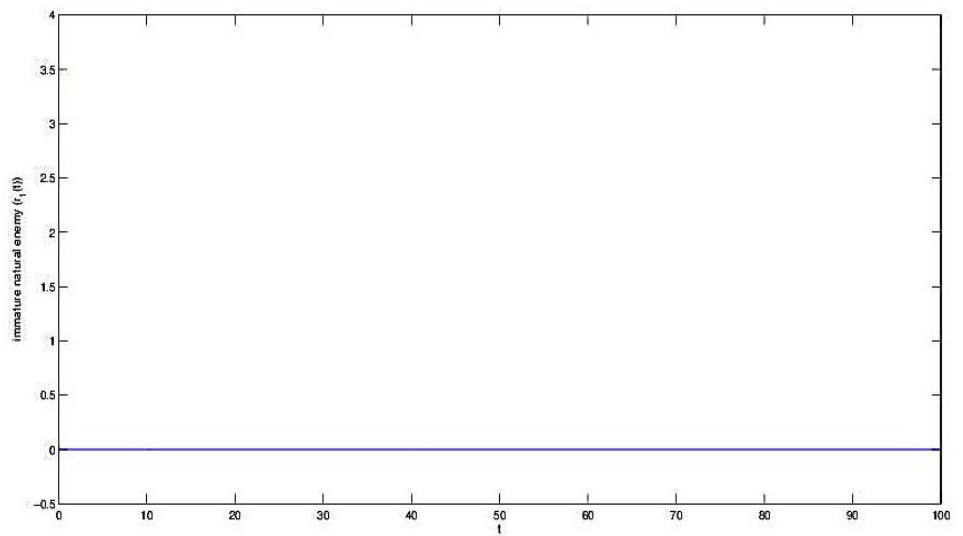

Figure 11. Immature natural enemy $\left(r_{1}(t)\right)$ vanishes for $\mu_{1}, \mu_{2}=0$

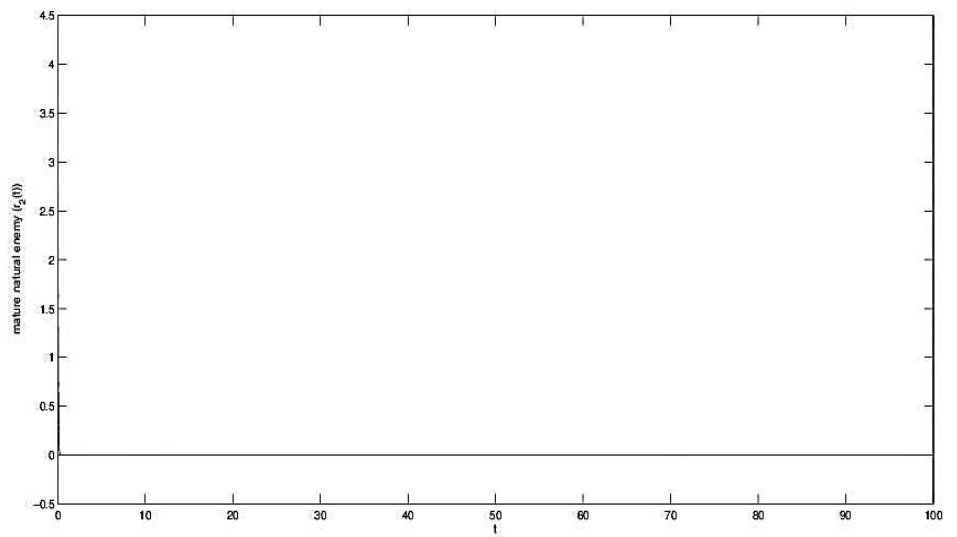

Figure 12. Mature natural enemy $\left(r_{2}(t)\right)$ for $\mu_{1}, \mu_{2}=0$

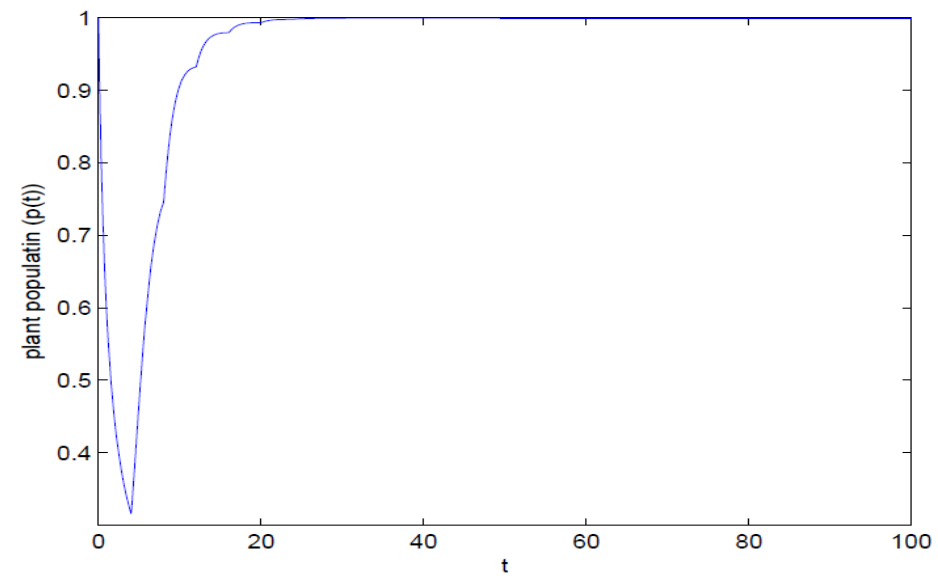

Figure 13. Plant population $(p(t))$ is stable for $\mu_{1}=100, \mu_{2}=50$ 
International Journal of Mathematical, Engineering and Management Sciences

Vol. 5, No. 4, 769-786, 2020

https://doi.org/10.33889/IJMEMS.2020.5.4.061

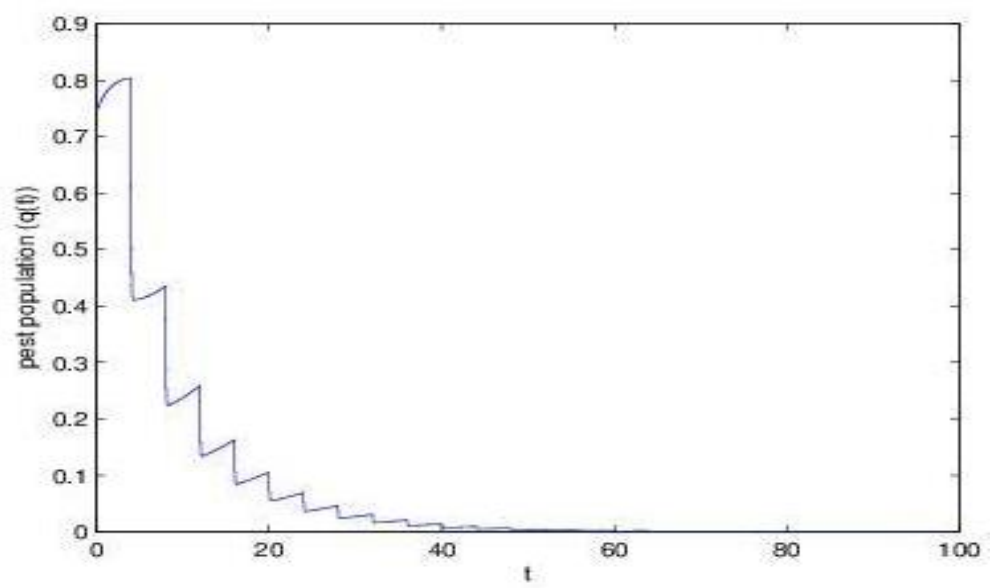

Figure 14. Pest population $(q(t))$ declines for $\mu_{1}=100, \mu_{2}=50$

\section{Conclusion}

In this paper, we have examine the effects of hybrid approach to control the pests by release of natural enemies and pesticides impulsively. It is evident that pest population can become extinct when large amount of the natural enemies are released impulsively. Thus, integrated pest management reduces pest quickly rather than using any one of the methods. Hence, in this paper, we have shown that by incorporating delay in the pests, we are able to control the pest population but to a lower threshold value which in a way is helpful as it is leading to early reduction in the pest which is not only economic but it also prevents pest resistance to crops. Incorporating delay lowered the threshold level from to $T_{\max }=7$ to $T_{\max }=0.8$ for the same set of parameters as in (Jatav \& Dhar, 2014). Thus, we can conclude that various control measures should be applied collectively for the eradication of pest. Such a practice improves economy as it is cost effective and synonymous with sustainable development.

\section{Conflicts of Interest}

The authors declare that there are no conflicts of interest regarding the publication of this paper.

\section{Acknowledgements}

The first author would like to express her sincere thanks to her guide, co-guide for their constant guidance and support and special thanks to all the reviewers and editor.

\section{References}

Akman, O., Comar, T.D., \& Hrozencik, D. (2015). On impulsive integrated pest management models with stochastic effects. Frontiers in Neuroscience, 9,119. doi: 10.3389/fnins.2015.00119.

Bainov, D.D., \& Simeonov, P. (1993). Impulsive differential equations: periodic solutions and application. Pitman Monographs and Surveys in Pure and Applied Mathematics, Chapman and Hall/CRC Press, ISBN 9780582096394. 
International Journal of Mathematical, Engineering and Management Sciences

Vol. 5, No. 4, 769-786, 2020

https://doi.org/10.33889/IJMEMS.2020.5.4.061

Changguo, L., Yongzhen, P., \& Xuehui, J. (2009). Dynamic behavior of a multiple species prey-predator system with impulsive chemical and biological control. In 2009 Fifth International Conference on Natural Computation (Vol. 5, pp. 477-481). IEEE. Tianjin, China.

El-Shafie, H. (2018). Integrated insect pest management. Pests - Insects, Management, Control [Working Title].

James, D.G. (1997). Imidacloprid increases egg production in Amblyseius victoriensis (Acari: Phytoseiidae). Experimental \& Applied Acarology, 21(2), 75-82.

Jatav, K.S., \& Dhar, J. (2014). Hybrid approach for pest control with impulsive releasing of natural enemies and chemical pesticides: a plant-pest-natural enemy model. Nonlinear Analysis: Hybrid Systems, 12, 79-92.

Jatav, K.S., Dhar, J., \& Nagar, A.K. (2014). Mathematical study of stage-structured pests control through impulsively released natural enemies with discrete and distributed delays. Applied Mathematics and Computation, 238, 511-526.

Liu, B., Xu, L., \& Kang, B. (2013). Dynamics of a stage structured pest control model in a polluted environment with pulse pollution input. Journal of Applied Mathematics, 2013. doi.org/10.1155/2013/678762.

Páez Chávez, J., Jungmann, D., \& Siegmund, S. (2018). A comparative study of integrated pest management strategies based on impulsive control. Journal of Biological Dynamics, 12(1), 318-341.

Song, Y., Wang, X., \& Jiang, W. (2014). The pest management model with impulsive control. Applied Mechanics and Materials, 519, 1299-1304. doi.org/10.4028/www.scientific.net/amm.

Tang, S., Xiao, Y., Chen, L., \& Cheke, R.A. (2005). Integrated pest management models and their dynamical behaviour. Bulletin of Mathematical Biology, 67(1), 115-135.

Yu, T., Tian, Y., Guo, H., \& Song, X. (2019). Dynamical analysis of an integrated pest management predatorprey model with weak Allee effect. Journal of Biological Dynamics, 13(1), 218-244.

Zhang, Y., Liu, B., \& Chen, L. (2004). Dynamical behavior of Volterra model with mutual interference concerning IPM. ESAIM: Mathematical Modelling and Numerical Analysis, 38(1), 143-155. 